\title{
A Case of Bleeding Pulmonary Artery Pseudoaneurysm Successfully Treated with Emergency Microsurgery
}

Yaso Saito, $\mathrm{MD}^{1} \quad$ Minoru Sakuraba, MD, $\mathrm{PhD}^{1} \quad$ Koreyuki Kurosawa, MD, $\mathrm{PhD}^{1}$ Tomoyuki Hishida, MD, PhD²

${ }^{1}$ Department of Plastic and Reconstructive Surgery, National Cancer Center Hospital East, Chiba, Japan

${ }^{2}$ Department of Respiratory Surgery, National Cancer Center Hospital East, Chiba, Japan
Address for correspondence Yaso Saito, MD, Department of Plastic and Reconstructive Surgery, National Cancer Center Hospital East, 6-5-1 Kashiwanoha, Kashiwa, Chiba, Japan 277-8577 (e-mail: 80saito@gmail.com).

J Reconstr Microsurg Open 2016;1:32-35.

\begin{abstract}
Pulmonary artery pseudoaneurysm (PAP) is rare. Reported causes of PAP include chest trauma, catheterization, and inflammatory diseases, such as pneumonia and lung abscess. We report a case of bleeding PAP successfully treated with emergency microsurgical reconstruction. A 50-year-old man with T3N1M0 lung cancer of the right upper lobe underwent right upper and middle lobectomy, inferior lobe $\mathrm{S} 6$ segmentectomy, and lymph node dissection. An infection of the right residual $\mathrm{S} 6$ developed. The inflammation then resulted in PAP. The infected S6 lesion was removed 6 weeks after the first surgery. However, 11 weeks after the first surgery the PAP bled. Although hemostasis was achieved through manual pressure, rebleeding was suspected, and surgery was emergently performed. Firm hemostasis was obtained after debridement and suturing of the lacerated pulmonary artery. A free rectus abdominis musculocutaneous (RAMC) flap with a $22 \times 10-\mathrm{cm}$ skin island was then raised and used to cover the pulmonary artery. The flap was used to fill the space that had been occupied by pyothorax. The patient was discharged from the hospital 19 days after the emergent

Keywords

- pulmonary artery pseudoaneurysm

- bronchopleural fistula

- emergent microsurgery microsurgery. The main treatment approaches for PAPs are catheter embolization and open surgery, but which approach is better is controversial. Surgical treatment was appropriate for our patient because the bleeding vessel was exposed after fenestration. An advantage of reconstruction with a free RAMC flap is the reduced risks of worsening infection and rebleeding, because the space of the defect is filled with tissue having an abundant blood supply and coverage is provided to the area of bleeding.
\end{abstract}

Pulmonary artery pseudoaneurysm (PAP) is a rare condition. Reported causes of PAP include chest trauma, catheterization, and inflammatory diseases, such as pneumonia and lung abscess. $^{1}$ We report a bleeding PAP successfully treated with emergency microsurgical reconstruction.

received

February 17, 2016

accepted after revision

April 3, 2016

published online

May 20, 2016 $10.1055 / \mathrm{s}-0036-1584222$ ISSN 2377-0813.

\section{Case Presentation}

A 50-year-old man with T3N1M0 lung cancer of the right upper lobe underwent right upper and middle lobectomy, inferior lobe S6 segmentectomy, and lymph node dissection. Dehiscence of

Copyright $\odot 2016$ by Thieme Medical Publishers, Inc., 333 Seventh Avenue, New York, NY 10001, USA.

License terms

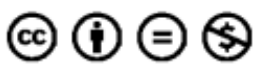


the bronchial anastomotic region occurred 9 days after surgery, and open-window thoracostomy was urgently performed 14 days after surgery. However, an infection of the right residual S6 developed and spread to the pulmonary artery (PA). Sputum culture 3 days after the first surgery had yielded Klebsiella pneumoniae (extended-spectrum $\beta$-lactamase-producing). The infected 66 lesion was removed 6 weeks after the first surgery. By 7 weeks after the first surgery, the intrathoracic infection had improved with oral levofloxacin and regular changing of the intrathoracic gauze ( $\mathbf{F i g} \cdot \mathbf{1 A}$ ). The patient was referred to our department for bronchopleural fistula (BPF) closure and chest wall reconstruction.

Four days before the planned reconstruction (11 weeks after the first surgery), bleeding was noted from the open-window space. Although hemostasis was achieved through manual pressure, microsurgery was emergently performed to treat a bleeding PAP that was suspected to have developed owing to long-term inflammation (- Fig. 1B, C). After debridement of the empyema space, the 2-cm-long lacerated portion of the PA was sutured with the surrounding connective tissue. A $2 \times 4-\mathrm{cm}$ fascial patch from the anterior sheath of the rectus abdominis muscle was used to cover the BPF. Firm hemostasis and the absence of air leakage were confirmed. A free rectus abdominis musculocutaneous (RAMC) flap with a $22 \times 10-\mathrm{cm}$ skin island was then harvested to cover the PA and fill the empyema space. The flap vessels were anastomosed with the right thoracoacromial artery (end-to-end, 9-0 nylon) and the right subclavian vein (end-to-side, 9-0 nylon; - Fig. 2).

The patient recovered quickly after surgery and was soon able to walk without supplemental oxygen. He was discharged 19 days after the emergent microsurgery. In the subsequent 2 years, there has been no recurrence of the PAP or the empyema following the BPF (-Fig. 3). The patient can walk continuously, if slowly, but has shortness of breath when climbing stairs.

\section{Discussion}

The present PAP bled 11 weeks after the first surgery. The exposure of the residual PA, as well as the right lung and other organs, to the focus of infection for 10 weeks after the dehiscence of the bronchial stump was the background upon which the PAP developed. Bao et al have reported a PAP following left upper sleeve lobectomy for a central squamous cell lung cancer. ${ }^{2}$ The PAP was attributed to both inflammation and iatrogenic PA trauma during lobectomy. Therefore, an operative procedure can be a contributing cause of PAP.

The main treatments for PAPs are catheter embolization and open surgery, but the choice of treatment is controversial. The main goals of treatment for empyema following BPF include calming down the infection, closing the BPF, and filling the dead space due to empyema. ${ }^{3,4}$ Additional goals in cases of PAP and empyema following BPF are rescuing the patient from hemorrhagic shock and preventing rebleeding. In the present case, we were able to achieve temporary hemostasis via manual pressure because of the thoracostomy. If manual pressure had not been possible, the treatment of first choice for rapid hemostasis might have been catheter coil

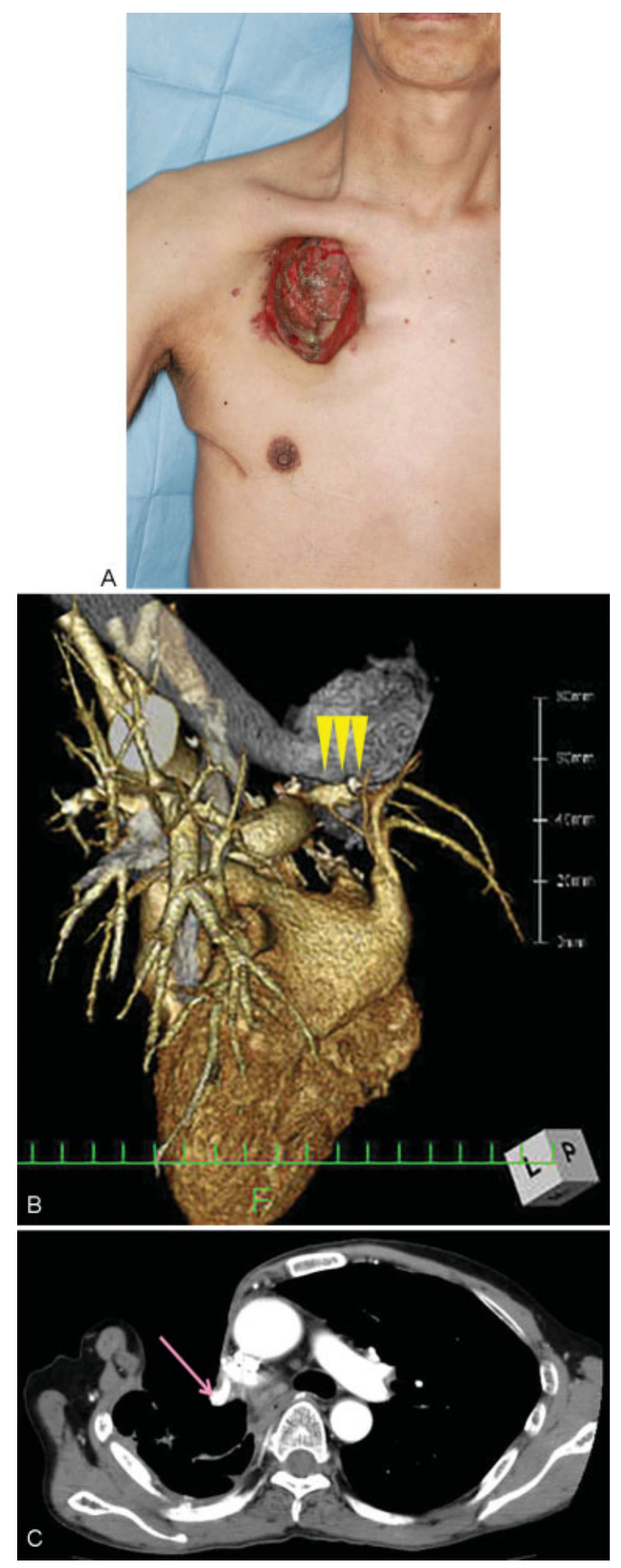

Fig. 1 (A) Preoperative photograph 2 weeks before the PAP bled. (B) 3D CT angiography. The lesion suspicious for PAP (arrowheads). (C) CT scans 3 days before the PAP bled. A mass which was suspicious for PAP was detected (arrow).

embolization. In retrospect, computed tomography (CT) before reconstruction had shown a possible unruptured PAP, for which coil embolization might be indicated. However, 

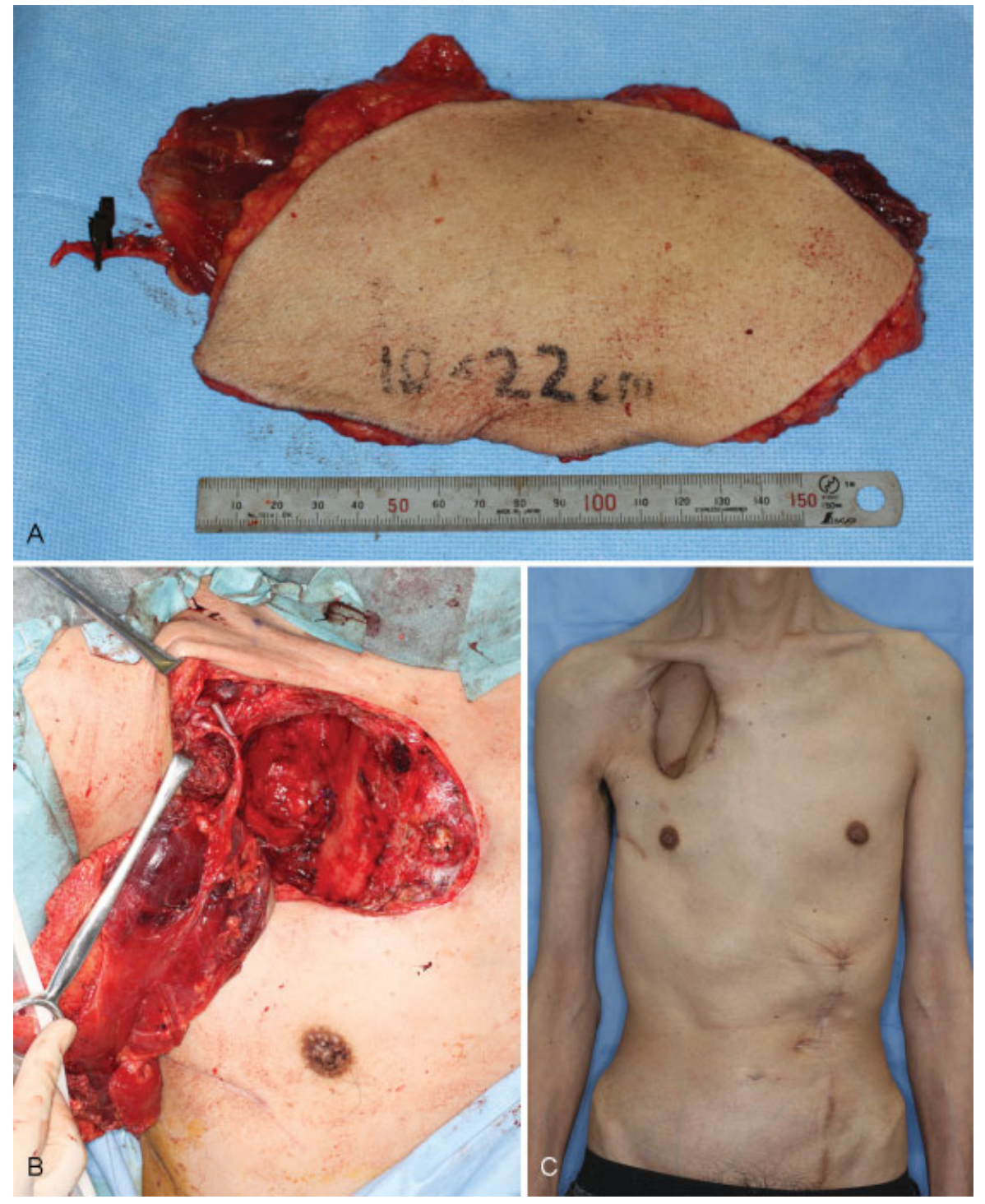

Fig. 2 (A) Intraoperative photograph. The harvested RAMC flap with $22 \times 10-\mathrm{cm}$ skin island. (B) After the anastomosis of flap vessels. (C) Postoperative photograph $\sim 2$ years after the reconstruction.

a disadvantage of coil embolization for PAP is the need for radical treatment of the BPF and empyema after hemostasis. Matsumura et al have reported a PAP after lung resection successfully treated with coil embolization. They believed

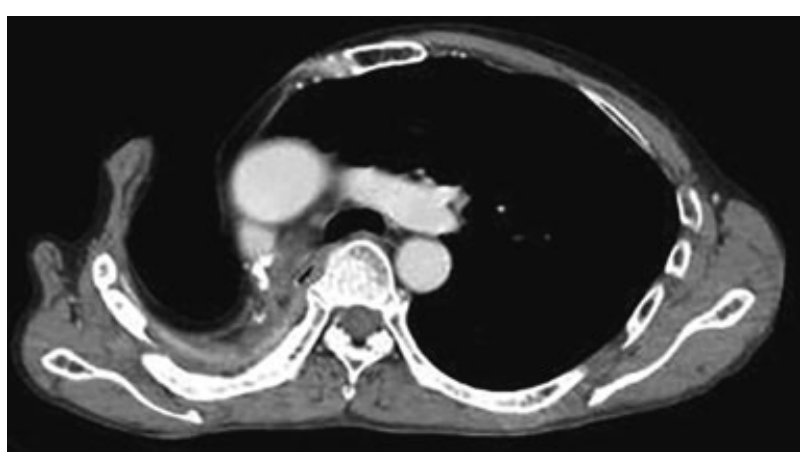

Fig. 3 CT image 2 years after the reconstructive surgery. There has been no recurrence of the PAP and empyema following BPF. that the limitations of this treatment are that it is symptomatic and that it might be followed by pulmonary infarction. ${ }^{5}$

Although reconstructive surgery with an RAMC free-flap transfer is more invasive than coil embolization, it has the great advantage of being able to be performed as one of a series of procedures: radical treatment for bleeding, reinforcement for BPF closure, filling of dead space, and preventing the spread of infection. According to Takanari et al, the advantages of free-flap transfer are that blood flow for infection control and volume for filling the intrathoracic space are obtained and that the flap is easy to inset into the space. ${ }^{6}$ Similar advantages were seen in the present case. Another reported advantage is that atrophy of the flap muscle allows the residual lung to expand after closure of the intrathoracic defect ${ }^{7}$; in the present case, however, expansion of the residual lung was not recognized in CT images 2 years after the first operation because most of the right lung had been resected (-Fig. 3). Finally, the greatest advantages of free-flap transfer in the present case were that the risk of 
rebleeding was reduced by a series of treatments beginning with the rescue from hemorrhagic shock and that the prognosis was improved.

\section{Conclusion}

Emergent microsurgery can be an effective treatment for bleeding PAP and empyema.

\section{References}

1 Lafita V, Borge MA, Demos TC. Pulmonary artery pseudoaneurysm: etiology, presentation, diagnosis, and treatment. Semin Intervent Radiol 2007;24(1):119-123
2 Bao M, Zhou Y, Jiang G, Chen C. Pulmonary artery pseudoaneurysm after a left upper sleeve lobectomy. World J Surg Oncol 2013;11:272

3 Walsh MD, Bruno AD, Onaitis MW, et al. The role of intrathoracic free flaps for chronic empyema. Ann Thorac Surg 2011;91(3): 865-868

4 Chen HC, Lo SJ, Kim JH. Management of intrathoracic defects. Semin Plast Surg 2011;25(1):70-77

5 Matsumura Y, Shiono S, Saito K, Sato T. Pulmonary artery pseudoaneurysm after lung resection successfully treated by coil embolization. Interact Cardiovasc Thorac Surg 2010;11(3):364-365

6 Takanari K, Kamei Y, Toriyama K, Yagi S, Torii S. Management of postpneumonectomy empyema using free flap and pedicled flap. Ann Thorac Surg 2010;89(1):321-323

7 Sakuraba M, Kimata Y, Uchiyama K, et al. Microvascular free flap reconstruction for empyema with bronchopleural fistula [Japanese]. J Jpn Soc Reconstr Microsurg 2000;13(1):37-42 